\title{
Physical variances in the hemolymph of Galleria mellonella L. (Lepidoptera: Pyralidae) following immune induction
}

\author{
Emad M.S. Barakat ${ }^{1}$, Shimaa.S.Ahmed ${ }^{1}$ and Mohamed O.Abokersh ${ }^{2}$ \\ 1- Department of Entomology, Faculty of Science, Ain Shams Univ., Abbassia, \\ Cairo, Egypt \\ 2- Department of Biology, Faculty of Science, Al-Asmarya Islamic Univ., Zeletin, Lybia
}

\begin{abstract}
The present study recorded the variations in the body water content and its relation to haemolymph volume, density and $\mathrm{pH}$ of the greater wax moth (Galleria mellonella) across different developmental stages at different time intervals post injection with Bacillus thuringiensis kurstaki. Preliminary results concerning Bt toxicity at a sub lethal concentration indicated that pupae were more resistant while, the adults were highly susceptible. Results concerning physiological investigations indicated that larvae had more body water content than pupae and adults, while haemolymph volume and density increased directly with insect development. Haemolymph $\mathrm{pH}$ had the same values in all developmental stages. Bt-injection decreased the body water content and, in the same time, increased the haemolymph volume, density and $\mathrm{pH}$ in all developmental stages. Maximum values attained at $48 \mathrm{~h}$ post injection. Pupae developed from previously treated larvae, and adults developed from previously challenged larvae and pupae showed significant changes compared with the normal ones and no changes as compared with treated insects. These findings show that different age groups respond to the impact of pathogenic bacteria by the same degree and the physiological changes did not affect the growth and transformation of this insect.
\end{abstract}

Key words: Galleria mellonella, Bacillus thuringiensis, water content, haemolymph volume, density and $\mathrm{pH}$.

\section{INTRODUCTION}

The economic importance of the greater wax moth, Galleria mellonella has led to a number of investigations on its life history, biology, behavior, ecology, physiology and control. However, an effective method of controlling this pest has not been developed. Physical and chemical methods are imperfect (Burges, 1978). Therefore, many studies have been conducted to find ways for controlling it. One new trend is applying microbial control agents, especially bacteria.

The association of bacteria with insects has been recorded since Pasteur's time and many species of pathogenic bacteria have been described. Since the description of Bacillus thuringiensis $\mathrm{Bt}$ by Berhelin (1986), perhaps more is known of its mechanism of pathogenicity than of any other invertebrate bacterial pathogen. Several authors reviewed the toxicity of this organism, in addition to the developmental studies by (Rogoff et al. 1969) which led to its use as a biological control agent for insect pests.

The high interest in biological means of controlling insects intensifies the need for investigating the response of insect to disease organisms and foreign proteins. The haemolymph, the tissue made up of fluids and different types of cells, offers a readily accessible criterion of this response. It can undergo quantitative changes to an extent virtually unknown for other tissues. Insect haemolymph is influenced at least on the level of its physical properties such as volume, density and $\mathrm{pH}$, or on its biochemical composition by several factors, among them are: diet, temperature and disease (Carrel et al., 1990), the physiological condition of the insect (Chapman, 2013), and the developmental stage (Jones, 1977). In addition, haemolymph serves as a major 
Emad M.S. Barakat et al.

compartment and storage reserve for water (Atmowidjojo et al., 1999). Changes in body water content did not attract the physiologists in the past, although it gives integrated picture with haemolymph about effect of treatment.

Due to the succeeded use of $\mathrm{Bt}$ against many lepidopteran crop pests, it was necessary to evaluate the use of this pathogen against G. mellonella. Furthermore, there are many physiological changes take place during the insect development, therefore, the response of insect to disease organisms are adapted to the specific needs of each developmental stage. In addition, the majority of the previous studies on this framework have been conducted on insect larvae and little or relatively no studies have been published comparing the changes induced in the haemolymph of larvae, pupae and adults of these insects. Therefore, this study was conducted to investigate and compare the effect of $\mathrm{Bt}$ challenge on the haemolymph of larvae, pupae and adults of $G$. mellonella, and also to determine the relevance of sex towards this challenge in the adult stage. More particularly, to detect if a previous challenge would enhance the same response of the later developmental stage, indicative of trans stadial transfer of response.

\section{MATERIALS AND METHODS Maintenance of insect:}

Galleria mellonella (L.) was collected and reared on an artificial diet, reported by Kulkarni et al. (2012). Last instar larvae, fresh pupae and adult moths (both sexes) on the day of their eclosion were used in the present investigation. They were collected and placed individually in small containers (approx. $13 \mathrm{~cm}^{3}$ ) for further development.

Preparation of the bacterial pathogen:

Bacillus thuringiensis kurstaki (Bt) as wettable powder formulation (AGERIN, $3200 \mathrm{IU} / \mathrm{mg}$ ) was grown aerobically at 28 $\pm 2{ }^{\circ} \mathrm{C}$ in nutrient broth tubes for $48 \mathrm{~h}$. Inoculates of the grown bacteria were cultured on nutrient agar plates at $28 \pm 2{ }^{\circ} \mathrm{C}$ for another $48 \mathrm{~h}$. Pure isolates were cultured on nutrient agar slants and incubated at $28 \pm 2^{\circ} \mathrm{C}$ for $48 \mathrm{~h}$, and then kept in the refrigerator at $4^{\circ} \mathrm{C}$ until used. Prior to use, pure isolates were grown on a nutrient agar medium at $28 \pm 2^{\circ} \mathrm{C}$ for $24 \mathrm{~h}$, harvested by suspending in a sterile distilled water and centrifuged at $6000 \mathrm{rpm}$ for $30 \mathrm{~min}$. The resultant sediment bacterial cells were washed three times with sterile distilled water and centrifuged again at the same rate till the solution becomes completely clear. The obtained bacterial pellet was then stored at $\varepsilon^{18^{\circ} \mathrm{C}}$ until required.

\section{Susceptibility levels to Pathogenic Agent:}

Bacterial suspension was adjusted to a concentration of $2.5 \times 10^{9}$ cells $/ \mathrm{ml}$ by the pour plate count method according to Campbell and Konowalchuk (1948).Different developmental stages of the G. mellonella were divided into three groups; normal insects (negative control without injection), control insects and bacterial injected insects. Larvae, pupae and adults of G. mellonella were injected with $2 \mu$ of each bacterial concentration; $2.5 \times 10^{3}, 2.5 \times 10^{4}, 2.5 \times 10^{5}, 2.5 \times 10^{6}$ and $2.5 \times 10^{7}$ cells $/ \mathrm{ml}$. Injection was made with a $10 \mu 1$ Hamilton micro-syringe fitted with a 26-gauge needle according to Miranpuri and Khachatourians (1993).

\section{Determination of the body weight and water content:}

Water content was determined gravimetrically for each individual larva, pupa and adult male and female as the difference between fresh (total) body weight to the nearest $0.10 \mathrm{mg}$ (wet weight) and the weight after drying for 2-4 $\mathrm{h}$ at $80^{\circ} \mathrm{C}$ till constant weight (dry weight). Normal insects and injected insects after3, 6, 12, 24 and $48 \mathrm{~h}$, along with control (water-injected) insects were used. They were weighed on electronic balance (METTLER, type BB300, Switzerland). 
Physical variances in the hemolymph of Galleria mellonella L.(Lepidoptera: Pyralidae) following immune induction

Determination of the haemolymph volume:

The haemolymph volume was determined for normal and injected insects at6, 12, 24 and $48 \mathrm{~h}$ post-injection, following the method described by Yeager and Munson (1950) and modified by Lee (1961). The tested insects were injected with $10 \mu \mathrm{l}$ of $0.2 \%$ amaranth red dye $(20$ $\mathrm{mg} / \mathrm{ml}$ of $0.5 \% \mathrm{NaCl}$ ). After allowing 5 min for the dye to mix thoroughly with the blood, $10 \mu \mathrm{l}$ of blood were extracted and diluted to $1 \mathrm{ml}$ with distilled water and mixed thoroughly to lyse the haemocytes and clear the sample. The absorbency was read by UNICO Spectrophotometer (SP2100 UV, China) at $515 \mathrm{~nm}$ using $1 \mathrm{ml}$ cuvette against the standard solution.

A series of saline solutions containing $0.05-0.3 \mathrm{mg}$ of amaranth dye were made to carry out a standard calibration curve. The absorbency of dyed solutions was measured at $515 \mathrm{~nm}$ against the blank prepared previously. The measurements were replicated 5 times. The weight of amaranth dye in unknown sample was calculated from the equation obtained from the standard calibration curve.

\section{Haemolymph density:}

The haemolymph densities of normal and injected insects at 6, 12, 24 and $48 \mathrm{~h}$ postinjection were determined according to the method described by Carrel et al. (1990). Haemolymph of the experimental insect was collected immediately into microcapillary tubes calibrated at $1 \mu \mathrm{l}$ and pre-weighed using an electronic balance (BRAINWEIGH B100, OHAUS Scale, CORP. USA). The filled tube was quickly reweighed. The haemolymph density was expressed as $\mathrm{mg} / \mu \mathrm{l}$.

\section{Haemolymph pH:}

The haemolymph $\mathrm{pH}$ was determined for normal and injected insects at $6,12,24$ and $48 \mathrm{~h}$ post-injection were determined according to the method described by Heimpel (1955). The bulb of the microelectrode (Model 671, pH meter, Extech, USA) was brought into contact with a drop of oozed haemolymph. All measurements were accomplished at $28 \pm$ $2^{\circ} \mathrm{C}$ and samples were replicated 5 times from each insect at each time intervals.

\section{Statistical analysis:}

Results of susceptibility tests were analyzed statistically by using software: Probit Analysis Program, Version 4.0. All the rest data were expressed as mean \pm standard error (SE) and analyzed by using the SPSS11.5.0 software (SPSS Inc.). The differences between means were analyzed by independent samples $t$-test and one-way ANOVA. The level of significance for each experiment was set at $\mathrm{P}<0.05$ or $\mathrm{P}<0.01$.

\section{RESULTS}

Susceptibility of $G$. mellonella to bacterial pathogen:

The estimated $\mathrm{LC}_{50}$ values, at $95 \%$ probability, were $2.5 \times 10^{4}, 6.7 \times 10^{4}$, and $3.5 \times 10^{3}$ cells $/ \mathrm{ml}$ for the larvae, pupae and adult insects, respectively (Table 1 ).

Table 1: Susceptibility of last instar larvae, pupae and adults of G. mellonella to B.t cells.

\begin{tabular}{|c|c|c|c|c|c|c|}
\hline $\begin{array}{c}\text { Concentration } \\
(\text { cells/ml) }\end{array}$ & \multicolumn{2}{|c|}{ Larval stage } & \multicolumn{2}{c|}{ Pupal stage } & \multicolumn{2}{c|}{ Adult stage } \\
\cline { 2 - 7 } & $\begin{array}{c}\text { Observed } \\
\text { mortality } \\
(\boldsymbol{\%})\end{array}$ & $\begin{array}{c}\text { Expected } \\
\text { mortality } \\
(\boldsymbol{\%})\end{array}$ & $\begin{array}{c}\text { Observed } \\
\text { mortality } \\
(\boldsymbol{\%})\end{array}$ & $\begin{array}{c}\text { Expected } \\
\text { mortality } \\
(\boldsymbol{\%})\end{array}$ & $\begin{array}{c}\text { Observed } \\
\text { mortality } \\
(\boldsymbol{\%})\end{array}$ & $\begin{array}{c}\text { Expected } \\
\text { mortality } \\
(\boldsymbol{\%})\end{array}$ \\
\hline $2.5 \times 10^{3}$ & 10 & 8.6 & 20 & 20.9 & 20 & 23.4 \\
\hline $2.5 \times 10^{4}$ & 20 & 27.2 & 40 & 38.2 & 40 & 45.6 \\
\hline $2.5 \times 10^{5}$ & 70 & 48.3 & 60 & 86.3 & 70 & 68.0 \\
\hline $2.5 \times 10^{6}$ & 80 & 74.3 & 90 & 90.5 & 90 & 91.3 \\
\hline Control & 0 & 0 & 0 & 0 & 0 & 0 \\
\hline Slope & \multicolumn{2}{|c|}{$0.7914 \pm 0.3365$} & $0.6689 \pm 0.1431$ & $0.7126 \pm 0.1983$ \\
\hline LC $_{\mathbf{5 0}}$ & \multicolumn{2}{|c|}{$2.5 \times 10^{4}$ cells/ml } & \multicolumn{2}{|c|}{$6.7 \times 10^{4}$ cells $/ \mathrm{ml}$} & $3.5 \times 10^{3} \mathrm{cells} / \mathrm{ml}$ \\
\hline
\end{tabular}


Emad M.S. Barakat et al.

Effects of Bt on body weight and water content of $G$. mellonella:

The body water content of un-injected

larvae was $180.68 \pm 2.09 \mathrm{mg}$. Bacterial injection decreased the values for the fresh body weight, dry body weight and water content significantly at $24 \mathrm{~h}(\mathrm{P}<0.05)$ and at

$48 \mathrm{~h}(\mathrm{P}<0.01)$ post-injection, but no changes were observed at 3,6 and $12 \mathrm{~h}$ post-injection $(\mathrm{P}$ $>0.05)$ as compared with water injected control larvae (Table 2)

Table 2: Total body weight, dry body weight and water content of $G$. mellonella (L.) larvae (last instar) determined at different time intervals post-injection with $B t$.

\begin{tabular}{|c|c|c|c|c|c|c|}
\hline \multirow{2}{*}{$\begin{array}{c}\text { Hours } \\
\text { post- } \\
\text { injection }\end{array}$} & \multicolumn{2}{|c|}{$\begin{array}{c}\text { Total body weight }(\mathbf{m g}) \\
\text { Mean } \pm \text { SE }\end{array}$} & \multicolumn{2}{c|}{$\begin{array}{c}\text { Dry body weight (mg) } \\
\text { Mean } \pm \text { SE }\end{array}$} & \multicolumn{2}{c|}{$\begin{array}{c}\text { Body water content (mg) } \\
\text { Mean } \pm \text { SE }\end{array}$} \\
\cline { 2 - 7 } & Control & Treated & Control & Treated & Control & Treated \\
\hline 3 & $249.33 \pm 1.93$ & $244.27 \pm 2.07$ & $70.40 \pm 1.92$ & $67.38 \pm 2.38$ & $178.93 \pm 2.79$ & $176.90 \pm 3.18$ \\
\hline 6 & $245.74 \pm 1.90$ & $241.81 \pm 1.81$ & $69.73 \pm 2.43$ & $66.12 \pm 1.80$ & $176.01 \pm 3.08$ & $175.69 \pm 1.14$ \\
\hline 12 & $244.98 \pm 2.36$ & $235.79 \pm 2.12$ & $68.59 \pm 2.47$ & $65.64 \pm 2.00$ & $176.39 \pm 3.11$ & $170.14 \pm 2.52$ \\
\hline 24 & $242.77 \pm 2.63$ & $227.26 \pm 2.32$ & $67.45 \pm 2.02$ & $60.60 \pm 1.61$ & $175.32 \pm 3.45$ & $166.66 \pm 2.77$ \\
\hline 48 & $239.33 \pm 2.81$ & $225.44 \pm 1.96$ & $65.89 \pm 2.05$ & $58.68 \pm 1.78$ & $173.44 \pm 4.09$ & $166.76 \pm 2.96$ \\
\hline $\begin{array}{c}\text { Un- } \\
\text { injected }\end{array}$ & \multicolumn{2}{|c|}{$246.88 \pm 1.92$} & \multicolumn{2}{|c|}{$73.48 \pm 1.77$} & & $180.68 \pm 2.09$ \\
\hline
\end{tabular}

$n=10$ insects per test.

Significat difference $(\mathrm{P}<0.05)$.

The body water content of the normal pupae was $128.99 \pm 3.73 \mathrm{mg}$. Following Bt injection, the whole parameters showed a

significant decrease $(\mathrm{P}<0.05)$ at $48 \mathrm{~h}$ postinjection (Table 3)

Table 3: Total body weight, dry body weight and water content of G. mellonella (L.) pupae determined at different time intervals post-injection with $B t$.

\begin{tabular}{|c|c|c|c|c|c|c|}
\hline \multirow{2}{*}{$\begin{array}{c}\text { Hours } \\
\text { postinjection }\end{array}$} & $\begin{array}{c}\text { Total body weight (mg) Mean } \\
\pm \text { SE }\end{array}$ & \multicolumn{2}{c|}{$\begin{array}{c}\text { Dry body weight }(\mathbf{m g}) \\
\text { Mean } \pm \text { SE }\end{array}$} & \multicolumn{2}{c|}{$\begin{array}{c}\text { Body water content } \\
\text { (mg) }\end{array}$} \\
& Control & Treated & Control & Treated & Control & Treated \\
\cline { 2 - 7 } & $181.70 \pm 2.46$ & $175.27 \pm 2.64$ & $51.15 \pm 1.89$ & $48.36 \pm 2.62$ & $130.55 \pm 3.06$ & $126.91 \pm 1.75$ \\
\hline 3 & $177.65 \pm 2.64$ & $171.31 \pm 1.64$ & $50.01 \pm 2.48$ & $46.41 \pm 2.08$ & $127.63 \pm 3.36$ & $124.90 \pm 2.28$ \\
\hline 6 & $175.92 \pm 2.76$ & $167.41 \pm 1.64$ & $48.88 \pm 2.47$ & $45.10 \pm 1.95$ & $127.03 \pm 4.32$ & $122.31 \pm 2.43$ \\
\hline 12 & $175.01 \pm 2.74$ & $163.46 \pm 1.86$ & $47.74 \pm 2.20$ & $42.64 \pm 1.88$ & $127.27 \pm 3.61$ & $120.82 \pm 2.90$ \\
\hline 24 & $171.24 \pm 1.50$ & $160.44 \pm 1.50$ & $45.11 \pm 1.93$ & $40.67 \pm 1.18$ & $126.13 \pm 1.99$ & $119.77 \pm 1.76$ \\
\hline 48 & $179.66 \pm 2.65$ & \multicolumn{3}{|c|}{$72.07 \pm 2.46$} & & $128.99 \pm 3.73$ \\
\hline Un-injected & \multicolumn{3}{|c|}{ Significant difference (P < 0.05$).$} \\
\hline
\end{tabular}

The body water content of the uninjected female adults was $89.23 \pm 2.28 \mathrm{mg}$. B.t-injection apparently does not affect the dry body weight at all time intervals, while the mean values of the wet weight and water

content showed a significant decrease $(\mathrm{P}<$ $0.05)$ at 24 and $48 \mathrm{~h}$ post-injection. No change was observed at 3,6 and $12 \mathrm{~h}$ after injection as compared to the water injected control insects (Table 4).

Table 4: Total body weight, dry body weight and water content of $G$. mellonella (L.) adult females determined at different time intervals post-injection with $B t$.

\begin{tabular}{|c|c|c|c|c|c|c|}
\hline \multirow[t]{2}{*}{$\begin{array}{c}\text { Hours } \\
\text { postinjection }\end{array}$} & \multicolumn{2}{|c|}{$\begin{array}{l}\text { Total body weight }(\mathrm{mg}) \\
\text { Mean } \pm \text { SE }\end{array}$} & \multicolumn{2}{|c|}{$\begin{array}{l}\text { Dry body weight }(\mathrm{mg}) \\
\text { Mean } \pm \text { SE }\end{array}$} & \multicolumn{2}{|c|}{$\begin{array}{l}\text { Body water content } \\
(\mathrm{mg}) \text { Mean } \pm \mathrm{SE}\end{array}$} \\
\hline & Control & Treated & Control & Treated & Control & Treated \\
\hline 3 & $139.02 \pm 2.00$ & $132.79 \pm 2.25$ & $48.28 \pm 1.68$ & $46.29 \pm 1.49$ & $90.74 \pm 3.12$ & $86.48 \pm 3.37$ \\
\hline 6 & $135.58 \pm 2.61$ & $128.42 \pm 2.32$ & $45.83 \pm 1.48$ & $43.38 \pm 1.62$ & $89.74 \pm 2.51$ & $85.05 \pm 2.28$ \\
\hline 12 & $133.12 \pm 1.96$ & $123.91 \pm 1.53$ & $43.83 \pm 1.68$ & $40.64 \pm 1.04$ & $89.30 \pm 1.53$ & $83.27 \pm 2.11$ \\
\hline 24 & $129.79 \pm 2.01$ & $118.91 \pm 1.72$ & $39.75 \pm 1.64$ & $36.55 \pm 1.24$ & $90.03 \pm 2.49$ & $82.36 \pm 2.47$ \\
\hline 48 & $126.85 \pm 1.87$ & $115.89 \pm 2.39$ & $37.84 \pm 2.53$ & $34.53 \pm 1.42$ & $89.01 \pm 3.53$ & $81.35 \pm 2.92$ \\
\hline Un-injected & \multicolumn{2}{|c|}{$135.19 \pm 1.98$} & \multicolumn{2}{|c|}{$45.96 \pm 2.14$} & \multicolumn{2}{|c|}{$89.23 \pm 2.28$} \\
\hline
\end{tabular}




\section{Physical variances in the hemolymph of Galleria mellonella L.(Lepidoptera: Pyralidae) following immune induction}

The body water content of the uninjected adult males was $72.84 \pm 4.39 \mathrm{mg}$. Injection of adult males with $B . t$ induced slight changes $(\mathrm{P}>0.05)$ at $6 \mathrm{~h}$ post injection in all studied parameters. A significant decrease $(\mathrm{P}<$
0.05) was observed in the fresh body weight and the water content at 12 and $24 \mathrm{~h}$, while a highly significant change $(\mathrm{P}<0.01)$ was noticed at $48 \mathrm{~h}$ after injection as compared with controls (Table 5)

Table 5: Total body weight, dry body weight and water content of $G$. mellonella (L.) adult males determined at different time intervals post-injection with $B t$.

\begin{tabular}{|c|c|c|c|c|c|c|}
\hline \multirow{2}{*}{$\begin{array}{c}\text { Hours } \\
\text { post- } \\
\text { injection }\end{array}$} & \multicolumn{2}{|c|}{$\begin{array}{c}\text { Total body weight }(\mathrm{mg}) \\
\text { Mean } \pm \text { SE } \\
\end{array}$} & \multicolumn{2}{|c|}{$\begin{array}{l}\text { Dry body weight }(\mathrm{mg}) \\
\text { Mean } \pm \text { SE } \\
\end{array}$} & \multicolumn{2}{|c|}{$\begin{array}{l}\text { Body water content } \\
(\mathrm{mg}) \text { Mean } \pm \mathrm{SE}\end{array}$} \\
\hline & Control & Treated & Control & Treated & Control & Treated \\
\hline 3 & $106.30 \pm 2.83$ & $102.49 \pm 1.91$ & $35.31 \pm 2.32$ & $33.44 \pm 1.73$ & $70.99 \pm 2.57$ & $69.05 \pm 2.58$ \\
\hline 6 & $102.62 \pm 1.95$ & $98.42 \pm 2.78$ & $32.79 \pm 1.69$ & $31.56 \pm 2.37$ & $69.34 \pm 1.51$ & $66.86 \pm 3.19$ \\
\hline 12 & $101.68 \pm 1.89$ & $91.52 \pm 2.69$ & $33.27 \pm 1.71$ & $29.37 \pm 2.41$ & $68.89 \pm 2.36$ & $62.15 \pm 3.77$ \\
\hline 24 & $99.26 \pm 2.93$ & $88.78 \pm 2.14$ & $30.10 \pm 2.38$ & $26.41 \pm 2.32$ & $69.16 \pm 3.49$ & $62.38 \pm 4.09$ \\
\hline 48 & $97.55 \pm 3.66$ & $84.65 \pm 2.89$ & $28.01 \pm 2.43$ & $23.47 \pm 2.45$ & $69.53 \pm 4.28$ & $61.18 \pm 4.04$ \\
\hline $\begin{array}{c}\text { Un- } \\
\text { injected }\end{array}$ & \multicolumn{2}{|c|}{$105.25 \pm 2.50$} & \multicolumn{2}{|c|}{$32.40 \pm 2.32$} & \multicolumn{2}{|c|}{$72.84 \pm 4.39$} \\
\hline
\end{tabular}

$n=10$ insects per test.

Pupae developed from challenged larvae were found to have a total body weight of $164.38 \pm 2.61 \mathrm{mg}$, dry body weight of $44.37 \pm$ $1.92 \mathrm{mg}$ and body water content of $120.01 \pm$ $2.38 \mathrm{mg}$ representing $73.01 \pm 4.2 \%$ of the insect fresh body weight. Adult developed from previously challenged larvae had a body weight that accounts for $102.56 \pm 2.65 \mathrm{mg}$, dry body weight of $34.29 \pm 1.11 \mathrm{mg}$ and water content $68.27 \pm 2.06 \mathrm{mg}$, representing $66.75 \%$ of insect fresh weight. Those developed from challenged pupae had $100 . .81 \pm 0.21 \mathrm{mg}$,
Significant difference $(\mathrm{P}<0.05)$.

$33.89 \pm 1.82 \mathrm{mg}$ and $66.92 \pm 2.62 \mathrm{mg}$ for total body weight, dry weight and water content, respectively. The water content account for $66.38 \%$ of total body weight. The total body weight and dry weight and water content of pupae coming from previously challenged larvae, and adults developed from treated larvae and pupae showed no significant changes $(\mathrm{P}>0.05)$ with treated insects, while it were significantly different $(\mathrm{P}<0.05)$ as compared with normal ones (Table 6)

Table 6: Total body weight, dry body weight and water content of $G$. mellonella (L.) pupae and adults developed from previously challenged stagesdetermined at different time intervals postinjection with $B t$.

\begin{tabular}{|c|l|c|c|c|}
\hline $\begin{array}{c}\text { Developmental } \\
\text { Stage }\end{array}$ & Treatment & $\begin{array}{c}\text { A }(\mathbf{m g}) \\
\text { Mean } \pm \text { SE }\end{array}$ & $\begin{array}{c}\text { Dry body weight } \\
(\mathbf{m g}) \text { Mean } \pm \text { SE }\end{array}$ & $\begin{array}{c}\text { Body water content } \\
(\mathbf{m g}) \text { Mean } \pm \text { SE }\end{array}$ \\
\hline \multirow{4}{*}{ Pupae } & Normal & $178 . .99 \pm 2.65 \mathrm{a}$ & $50.00 \pm 2.4 \mathrm{a}$ & $128.99 \pm 3.73 \mathrm{a}$ \\
\cline { 2 - 5 } & Treated & $160.44 \pm 1.50 \mathrm{~b}$ & $42.74 \pm 1.18 \mathrm{~b}$ & $117.70 \pm 1.76 \mathrm{~b}$ \\
\cline { 2 - 5 } & $\begin{array}{l}\text { Developed from } \\
\text { treated larva }\end{array}$ & $164 . .38 \pm 2.61 \mathrm{~b}$ & $44.37 \pm 1.92 \mathrm{a}$ & $120.01 \pm 2.38 \mathrm{~b}$ \\
\hline \multirow{4}{*}{ Adults } & Normal & $116.41 \pm 2.62 \mathrm{a}$ & $40.60 \pm 1.91 \mathrm{a}$ & $75.81 \pm 2.08 \mathrm{a}$ \\
\cline { 2 - 5 } & Treated & $97.28 \pm 3.01 \mathrm{~b}$ & $32.00 \pm 2.12 \mathrm{~b}$ & $65.28 \pm 1.39 \mathrm{~b}$ \\
\cline { 2 - 5 } & $\begin{array}{l}\text { Developed from } \\
\text { treated larva }\end{array}$ & $102.56 \pm 2.65 \mathrm{~b}$ & $34.29 \pm 1.11 \mathrm{~b}$ & $68.27 \pm 2.06 \mathrm{~b}$ \\
\cline { 2 - 5 } & $\begin{array}{l}\text { Developed from } \\
\text { treated pupa }\end{array}$ & $100 . .81 \pm 0.21 \mathrm{~b}$ & $33.89 \pm 1.82 \mathrm{~b}$ & $66.92 \pm 2.62 \mathrm{~b}$ \\
\hline
\end{tabular}

Different letters for each stage indicate significant change $(\mathrm{P}<0.05)$. 
Emad M.S. Barakat et al.

Effect of Bt on haemolymph volume of G. mellonella:

The normal (un-injected) larvae, pupae, adult females and adult males of $G$. mellonella contained 89.79 $\pm 2.19,43.27 \pm$ $2.76,16.06 \pm 2.47$ and $11.12 \pm 2.11 \mu 1$ of haemolymph per insect, respectively. These values contributed to about 49.70, $33.55,18.00$ and $16.88 \%$ of the total water content of the larva pupae, adult

Table 7: Haemolymph volume ( $\mu \mathrm{l} /$ larva) of $G$. mellonella larvae, pupae, adult females and adult males determined at different time intervals post-injection with $B t$ using amaranth dye method

\begin{tabular}{|c|c|c|c|c|c|c|c|c|}
\hline \multirow{3}{*}{$\begin{array}{c}\text { Hours } \\
\text { post } \\
\text { injection }\end{array}$} & \multicolumn{8}{|c|}{ Haemolymph volume $(\mu \mathrm{l} /$ larva) $($ Mean $\pm \mathrm{SE})$} \\
\hline & \multicolumn{2}{|c|}{ Larval stage } & \multicolumn{2}{|c|}{ Pupal stage } & \multicolumn{2}{|c|}{ Adult females } & \multicolumn{2}{|c|}{ Adult males } \\
\hline & Control & Treated & Control & Treated & Control & Treated & Control & Treated \\
\hline 6 & $\begin{array}{c}94.21 \\
\pm 2.40\end{array}$ & $\begin{array}{c}116.82 \pm \\
1.38\end{array}$ & $\begin{array}{c}52.81 \\
\pm 2.53\end{array}$ & $\begin{array}{r}68.94 \\
\pm 1.53\end{array}$ & $\begin{array}{r}22.01 \\
\pm 2.43 \\
\end{array}$ & $\begin{array}{r}34.99 \\
\pm 1.61\end{array}$ & $\begin{array}{r}16.18 \\
\pm 2.68\end{array}$ & $\begin{array}{r}28.82 \\
\pm 2.29 \\
\end{array}$ \\
\hline 12 & $\begin{array}{r}95.01 \\
\pm 2.98\end{array}$ & $\begin{array}{c}122.14 \pm \\
1.63\end{array}$ & $\begin{array}{l}55.01 \\
\pm 2.29\end{array}$ & $\begin{array}{c}69.81 \\
\pm 2.16\end{array}$ & $\begin{array}{r}17.47 \\
\pm 2.45\end{array}$ & $\begin{array}{c}3798 \\
\pm 0.95\end{array}$ & $\begin{array}{r}13.09 \\
\pm 1.71\end{array}$ & $\begin{array}{r}32.14 \\
\pm 1.51\end{array}$ \\
\hline 24 & $\begin{array}{r}1.67 \\
\pm 2.69 \\
\end{array}$ & $\begin{array}{c}120.08 \pm \\
1.55\end{array}$ & $\begin{array}{r}51.07 \\
\pm 2.86 \\
\end{array}$ & $\begin{array}{r}70.45 \\
\pm 2.90 \\
\end{array}$ & $\begin{array}{r}15.12 \\
\pm 1.61 \\
\end{array}$ & $\begin{array}{c}37.14 \\
\pm 0.95 \\
\end{array}$ & $\begin{array}{r}10.25 \\
\pm 2.44 \\
\end{array}$ & $\begin{array}{r}29.84 \\
\pm 2.51 \\
\end{array}$ \\
\hline 48 & $\begin{array}{c}86.61 \\
\pm 2.95 \\
\end{array}$ & $\begin{array}{c}115.41 \pm \\
1.49\end{array}$ & $\begin{array}{l}47.84 \\
\pm 2.21 \\
\end{array}$ & $\begin{array}{r}66.77 \\
\pm 1.37 \\
\end{array}$ & $\begin{array}{l}15.91 \\
\pm 2.33 \\
\end{array}$ & $\begin{array}{c}31.92 \\
\pm 2.84\end{array}$ & $\begin{array}{c}8.12 \\
\pm 2.22 \\
\end{array}$ & $\begin{array}{r}27.31 \\
\pm 2.32 \\
\end{array}$ \\
\hline $\begin{array}{c}\text { Un- } \\
\text { injected }\end{array}$ & \multicolumn{2}{|c|}{$89.79 \pm 2.19$} & \multicolumn{2}{|c|}{$43.27 \pm 2.76$} & \multicolumn{2}{|c|}{$16.06 \pm 2.47$} & \multicolumn{2}{|c|}{$11.12 \pm 2.11$} \\
\hline
\end{tabular}

Significant difference $(\mathrm{P}<0.05)$.

Pupae developed from challenged larvae were found to have about $59.91 \pm$ $2.09 \mu 1$ of haemolymph/pupa. This value contributed to about $49.92 \%$ of the total water content of these insects and about $36.45 \%$ of the fresh body weight. Continuously, adults developed from these challenged larvae had $25.12 \pm 0.87 \mu \mathrm{l}$ of haemolymph/insect. This account for $36.80 \%$ of total body water content and about $24.49 \%$ of the wet body weight, while those developed from previously

Table 8: Haemolymph volume ( $\mu \mathrm{l}$ / insect) of $G$. mellonella pupae and adults developed from previously challenged stages, using amaranth dye method.

\begin{tabular}{|c|l|c|}
\hline Developmental Stage & \multicolumn{1}{|c|}{ Treatment } & $\begin{array}{c}\text { Haemolymph volume }(\boldsymbol{\mu l} / \text { larva) } \\
\text { (Mean } \pm \text { SE) }\end{array}$ \\
\hline \multirow{4}{*}{ Pupae } & Normal & $43.27 \pm 2.76 \mathrm{a}$ \\
\cline { 2 - 3 } & Treated & $66.77 \pm 1.37 \mathrm{~b}$ \\
\cline { 2 - 3 } & Developed from treated larva & $59.91 \pm 2.09 \mathrm{~b}$ \\
\hline & Normal & $14.51 \pm 1.34 \mathrm{a}$ \\
\hline \multirow{3}{*}{ Adults } & Treated & $30.12 \pm 2.71 \mathrm{~b}$ \\
\cline { 2 - 3 } & Developed from treated larva & $25.12 \pm 0.87 \mathrm{~b}$ \\
\cline { 2 - 3 } & Developed from treated pupa & $24.76 \pm 1.43 \mathrm{~b}$ \\
\hline
\end{tabular}

Different letters for each stage indicate significant change $(\mathrm{P}<0.05)$. females and adult males, respectively, and about 36.51, 24.17, 11.88 and $11.11 \%$ of their total body weights, respectively. After bacterial treatment, haemolymph volumes of larvae, pupae, adult females and adult males showed significant increase $(\mathrm{P}<0.05)$ at different time intervals as compared to the water injected controls (Table 7).

challenged pupae account had $24.76 \pm$ $1.43 \mu \mathrm{l}$ of haemolymph/insect, constituting about $37.00 \%$ of the water content and about $24.56 \%$ of the total fresh weight. The haemolymph volume of pupae coming from previously challenged larvae, and adults developed from treated larvae and pupae showed no significant changes $(\mathrm{P}>$ $0.05)$ with treated insects, while it significantly increase $(\mathrm{P}<0.05)$ as compared with normal ones (Table 8 ) 
Effect of Bt injection on haemolymph density of $G$. mellonella:

The estimated mean values of the haemolymph density of un-injected larvae, pupae, adult females and adult males of $G$. mellonella were $0.98 \pm 0.03,1.04 \pm 0.02$, $1.03 \pm 0.03$ and $1.01 \pm 0.02 \mathrm{mg} / \mu \mathrm{l}$, respectively. In bacterial injected insects, the haemolymph density increased significantly $(\mathrm{P}<0.05)$ only at 6,12 and $24 \mathrm{~h}$, and then decreased to the original level at $48 \mathrm{~h}$ (Table 9).

Table 9: Haemolymph density (mg/ $\mu \mathrm{l})$ of $G$. mellonella larvae, pupae, adult females and adult males determined at different time intervals post-injection with $\mathrm{Bt}$

\begin{tabular}{|c|c|c|c|c|c|c|c|c|}
\hline \multirow{3}{*}{$\begin{array}{c}\text { Hours } \\
\text { post } \\
\text { injection }\end{array}$} & \multicolumn{8}{|c|}{ Haemolymph density $(\mathrm{mg} / \mu \mathrm{l})($ Mean \pm SE) } \\
\hline & \multicolumn{2}{|c|}{ Larval stage } & \multicolumn{2}{|c|}{ Pupal stage } & \multicolumn{2}{|c|}{ Adult females } & \multicolumn{2}{|c|}{ Adult males } \\
\hline & Control & Treated & Control & Treated & Control & Treated & Control & Treated \\
\hline 6 & $\begin{array}{c}0.976 \pm \\
0.01\end{array}$ & $\begin{array}{c}1.27 \pm \\
0.02\end{array}$ & $\begin{array}{c}1.02 \pm \\
0.03\end{array}$ & $\begin{array}{c}1.44 \pm \\
0.05\end{array}$ & $\begin{array}{c}1.04 \pm \\
0.02\end{array}$ & $\begin{array}{c}1.34 \pm \\
0.03\end{array}$ & $\begin{array}{c}0.973 \pm \\
0.02\end{array}$ & $\begin{array}{c}1.23 \pm \\
0.02\end{array}$ \\
\hline 12 & $\begin{array}{c}1.05 \pm \\
0.02\end{array}$ & $\begin{array}{c}1.19 \pm \\
0.03\end{array}$ & $\begin{array}{c}0.99 \pm \\
0.01\end{array}$ & $\begin{array}{l}1.47 \pm \\
0.05\end{array}$ & $\begin{array}{c}1.14 \pm \\
0.04\end{array}$ & $\begin{array}{c}1.22 \pm \\
0.04\end{array}$ & $\begin{array}{c}1.03 \pm \\
0.03\end{array}$ & $\begin{array}{c}1.18 \pm \\
0.02\end{array}$ \\
\hline 24 & $\begin{array}{c}0.94 \pm \\
0.02\end{array}$ & $\begin{array}{c}1.06 \pm \\
0.03\end{array}$ & $\begin{array}{c}1.00 \pm \\
0.03\end{array}$ & $\begin{array}{c}1.35 \pm \\
0.06\end{array}$ & $\begin{array}{c}0.98 \pm \\
0.02\end{array}$ & $\begin{array}{c}1.31 \pm \\
0.03\end{array}$ & $\begin{array}{c}1.06 \pm \\
0.03\end{array}$ & $\begin{array}{c}1.53 \pm \\
0.04\end{array}$ \\
\hline 48 & $\begin{array}{c}1.01 \pm \\
0.01\end{array}$ & $\begin{array}{c}1.08 \pm \\
0.03\end{array}$ & $\begin{array}{c}1.01 \pm \\
0.03\end{array}$ & $\begin{array}{c}1.33 \pm \\
0.04\end{array}$ & $\begin{array}{c}0.99 \pm \\
0.03\end{array}$ & $\begin{array}{c}1.38 \pm \\
0.03\end{array}$ & $\begin{array}{c}0.98 \pm \\
0.02\end{array}$ & $\begin{array}{c}1.32 \pm \\
0.03\end{array}$ \\
\hline $\begin{array}{c}\text { Un- } \\
\text { injected }\end{array}$ & \multicolumn{2}{|c|}{$0.98 \pm 0.03$} & \multicolumn{2}{|c|}{$1.04 \pm 0.02$} & \multicolumn{2}{|c|}{$1.03 \pm 0.03$} & \multicolumn{2}{|c|}{$1.01 \pm 0.02$} \\
\hline
\end{tabular}

Significat difference $(\mathrm{P}<0.05)$.

Table 10: Haemolymph density $(\mathrm{mg} / \mu \mathrm{l})$ of $G$. mellonella pupae and adults developed from previously challenged stages

\begin{tabular}{|c|l|c|}
\hline Developmental Stage & \multicolumn{1}{|c|}{ Treatment } & $\begin{array}{c}\text { Haemolymph density }(\mathbf{m g} / \boldsymbol{\mu l})(\mathbf{M e a n} \\
\mathbf{\mathbf { S S E }})\end{array}$ \\
\hline \multirow{3}{*}{ Pupae } & Normal & $1.04 \pm 0.02 \mathrm{a}$ \\
\cline { 2 - 3 } & Treated & $1.38 \pm 0.03 \mathrm{~b}$ \\
\cline { 2 - 3 } & Developed from treated larva & $1.44 \pm 0.04 \mathrm{~b}$ \\
\hline \multirow{4}{*}{ Adults } & Normal & $1.02 \pm 0.03 \mathrm{a}$ \\
\cline { 2 - 3 } & Treated & $1.35 \pm 0.04 \mathrm{~b}$ \\
\cline { 2 - 3 } & Developed from treated larva & $1.33 \pm 0.04 \mathrm{~b}$ \\
\cline { 2 - 3 } & Developed from treated pupa & $1.28 \pm 0.03 \mathrm{~b}$ \\
\hline
\end{tabular}

Different letters for each stage indicate significant change $(\mathrm{P}<0.05)$.

Effect of Bt on haemolymph pH of $G$. mellonella:

Table (11) indicated that the mean haemolymph $\mathrm{pH}$ values of un-injected larvae, pupae, adult females and adult males of $G$. mellonella were $7.13 \pm 0.03$, $7.10 \pm 0.04,7.08 \pm 0.03$ and $7.10 \pm 0.04$, respectively (i.e., slightly alkaline haemolymph). Although, there were significant increases $(P<0.05)$ at 6,12 and $24 \mathrm{~h}$ in the haemolymph $\mathrm{pH}$ of Bttreated insects, the haemolymph $\mathrm{pH}$ was still slightly alkaline. 
Emad M.S. Barakat et al.

Table 11: Haemolymph pH of G. mellonella larvae, pupae, adult females and adult males determined at different time intervals post-injection with $\mathrm{Bt}$

\begin{tabular}{|c|c|c|c|c|c|c|c|c|}
\hline \multirow{3}{*}{$\begin{array}{c}\text { Hours } \\
\text { post } \\
\text { injection }\end{array}$} & \multicolumn{8}{|c|}{ Haemolymph pH (Mean \pm SE) } \\
\hline & \multicolumn{2}{|c|}{ Larval stage } & \multicolumn{2}{|c|}{ Pupal stage } & \multicolumn{2}{|c|}{ Adult females } & \multicolumn{2}{|c|}{ Adult males } \\
\hline & Control & Treated & Control & Treated & Control & Treated & Control & Treated \\
\hline 6 & $\begin{array}{c}7.03 \pm \\
0.02\end{array}$ & $\begin{array}{c}7.30 \pm \\
0.01\end{array}$ & $\begin{array}{c}6.90 \pm \\
0.06^{*}\end{array}$ & $\begin{array}{c}6.80 \pm \\
0.02\end{array}$ & $\begin{array}{c}7.05 \pm \\
0.02\end{array}$ & $\begin{array}{c}7.10 \pm \\
0.04\end{array}$ & $\begin{array}{c}7.01 \pm \\
0.04\end{array}$ & $\begin{array}{c}7.10 \pm \\
0.04\end{array}$ \\
\hline 12 & $\begin{array}{c}7.03 \pm \\
0.02\end{array}$ & $\begin{array}{c}7.12 \pm \\
0.01\end{array}$ & $\begin{array}{c}6.81 \pm \\
0.04\end{array}$ & $\begin{array}{c}6.99 \pm \\
0.02\end{array}$ & $\begin{array}{c}7.02 \pm \\
0.02\end{array}$ & $\begin{array}{c}7.10 \pm \\
0.04\end{array}$ & $\begin{array}{c}7.06 \pm \\
0.02\end{array}$ & $\begin{array}{c}7.14 \pm \\
0.04\end{array}$ \\
\hline 24 & $\begin{array}{c}7.06 \pm \\
0.02\end{array}$ & $\begin{array}{l}7.16 \pm \\
0.02\end{array}$ & $\begin{array}{l}7.09 \pm \\
0.03\end{array}$ & $\begin{array}{c}7.14 \pm \\
0.02\end{array}$ & $\begin{array}{c}7.07 \pm \\
0.03\end{array}$ & $\begin{array}{c}7.14 \pm \\
0.04\end{array}$ & $\begin{array}{c}7.04 \pm \\
0.02\end{array}$ & $\begin{array}{c}7.16 \pm \\
0.04\end{array}$ \\
\hline 48 & $\begin{array}{c}7.09 \pm \\
0.03\end{array}$ & $\begin{array}{c}7.11 \pm \\
0.02\end{array}$ & $\begin{array}{c}7.03 \pm \\
0.03\end{array}$ & $\begin{array}{c}7.17 \pm \\
0.02\end{array}$ & $\begin{array}{c}7.10 \pm \\
0.04\end{array}$ & $\begin{array}{c}7.14 \pm \\
0.04\end{array}$ & $\begin{array}{c}7.08 \pm \\
0.03\end{array}$ & $\begin{array}{c}7.13 \pm \\
0.04\end{array}$ \\
\hline $\begin{array}{c}\text { Un- } \\
\text { injected }\end{array}$ & \multicolumn{2}{|c|}{$7.13 \pm 0.03$} & \multicolumn{2}{|c|}{$7.10 \pm 0.04$} & \multicolumn{2}{|c|}{$7.08 \pm 0.03$} & \multicolumn{2}{|c|}{$7.10 \pm 0.04$} \\
\hline
\end{tabular}

Significant difference $(\mathrm{P}<0.05)$.

The haemolymph mean $\mathrm{pH}$ value of pupae developed from challenged larvae were found to be $7.15 \pm 0.02$, and adults developed from these larvae had $\mathrm{pH}$ value of $7.14 \pm 0.02$, while those developed from previously challenged pupae account 7.13

Table 12: Haemolymph $\mathrm{pH}$ of $G$. mellonella pupae and adults developed from previously challenged stages, using amaranth dye method

\begin{tabular}{|c|l|c|}
\hline Developmental Stage & \multicolumn{1}{|c|}{ Treatment } & Haemolymph pH (Mean \pm SE) \\
\hline \multirow{3}{*}{ Pupae } & Normal & $7.10 \pm 0.04 \mathrm{a}$ \\
\cline { 2 - 3 } & Treated & $7.17 \pm 0.02 \mathrm{~b}$ \\
\cline { 2 - 3 } & $\begin{array}{l}\text { Developed from } \\
\text { treated larva }\end{array}$ & $7.15 \pm 0.02 \mathrm{~b}$ \\
\hline \multirow{5}{*}{ Adults } & Normal & $7.09 \pm 0.02 \mathrm{a}$ \\
\cline { 2 - 3 } & Treated & $7.16 \pm 0.02 \mathrm{~b}$ \\
\cline { 2 - 3 } & $\begin{array}{l}\text { Developed from } \\
\text { treated larva }\end{array}$ & $7.14 \pm 0.02 \mathrm{~b}$ \\
\cline { 2 - 3 } & $\begin{array}{l}\text { Developed from } \\
\text { treated pupa }\end{array}$ & $7.13 \pm 0.02 \mathrm{~b}$ \\
\hline
\end{tabular}

Different letters for each stage indicate significant change $(\mathrm{P}<0.05)$.

\section{DISCUSSION}

Not only $G$. mellonella are important to apiculture industry, but also they are investigated considerably more as a model organism for studying insect physiology. This comes from the fact that they can be easily reared and maintained in large numbers. There are no ethical constraints and their short life cycle makes them ideal for large-scale studies. Given the size of the insect, it is possible to obtain easily hemolymph and other tissues (Wojda, 2017). Many studies have \pm 0.02 . The haemolymph $\mathrm{pH}$ of pupae coming from previously challenged larvae, and adults developed from treated larvae and pupae showed no significant changes $(\mathrm{P}>0.05)$ with treated insects (Table 12). performed using insects as an alternative model host for investigating virulence factors of human pathogenic bacteria (Scully and Bidochka, 2006; Lionakis, 2011), and this substitution has several benefits. Within this frame, G. mellonella are used in our study to answer several questions; the most important are; (1) what are the physiological changes, due to infection, that take place during the insect development, (2) what is the developmental stage of an insect that has more susceptibility to microbial invasion, 


\section{Physical variances in the hemolymph of Galleria mellonella L.(Lepidoptera: Pyralidae) following immune induction}

(3) is the sex has a relevance towards this response, and finally (4) is a previous challenge would enhance the same response of the later developmental stage?

Bacterial formulations, especially $\mathrm{Bt}$ are receiving more attention as microbial insecticides and also are being identified as key natural mortality factors in the environment of many important insect pests. It is active against many pest species including the greater wax moth (Bravo et al., 2005). However, lack of information on the quantity of test pathogen that reach to the test insect makes comparison of the infectivity of different or the same pathogen, extremely difficult. This in turn would lead to difficulty in the standardization of insect pathogenic micro-organisms, and hence also in the evaluation of their potential in insect control. This factor has attracted considerable attention in recent times, to quantify the actual amount of pathogen introduced into the tested insect. Therefore, the pathogen in the present study was placed in direct contact with the susceptible tissue (hemocoel) by injection, so that variation induced by loss of dose and irregularities of invasion was avoided (Barakat, 2001). Several workers intended to use this technique especially for the study of insect immunity and the observation of the biochemical and physiological changes induced by pathogenic infection. Among the numerous authors who have used the same technique are: Meshrifand Barakat (2002); Krishnedu et al. (2010); Mo'men et al. (2012); Mahmoud et al. (2015); Pereira et al. (2015) using different insects and different bacterial pathogens.

Based on qualitative descriptions on how different developmental stages of G. mellonella suffered from infection with the chosen bacteria and the resulting mortality patterns, the present study clearly demonstrated that the pupae of are more resistant to $\mathrm{Bt}\left(\mathrm{LC}_{50}=5.9 \times 10^{5}\right.$ cells $/ \mathrm{ml})$. In addition, the presence of proteases and other metabolic compounds, due to the degradation of larval tissues, will add to a bacteria unfriendly environment. Similar explanation was given by Kurata et al. (1992); Natori et al. (1999) who attributed the high level of resistance found in pupae, against bacteria to the presence of defense proteins that function within development and expressed during the pupal stage. Also the present study stated that the adult resistance was found to be the least effective $\left(\mathrm{LC}_{50}=4.6 \times 10^{4}\right.$ cells $\left./ \mathrm{ml}\right)$. This may be resulted from that, the adult immune defense system is traded off against some other fitness components as survival and reproduction. This observation was confirmed by some studies (Sheldon and Verhulst 1996; Schmid-Hempel 2003 a \& 2005).

Inter- and intra-specific variation in pathogenicity may be attributed to secondary metabolites and/or enzymes, bacteria secret into their hosts.Such compounds have been suggested to be important virulence factors allowing pathogens to successfully invade hosts (Meshrif et al., 2007).

Typically, the insect responses to the infection on protein distribution, as well as on water distribution. It was considered that the results for blood volume would be more informative with knowledge of the dry weight and water values for comparison. Desiccations were carried out concomitant with those on microbial variation (Barakatand Meshrif, 2007). Results are expressed as percentages of the total weight, thus permitting comparisons of relative values from individuals of different weight during development.

The present results indicated that the estimated percentage of body water content of different developmental stages of Galleria ranges from more than $50 \%$ to less than $90 \%$ of the total body weight. These results are supported by the work of 


\section{Emad M.S. Barakat et al.}

Wigglesworth (1974) on different insect species from different orders.

Changes in the body weight attracted the immunologists in the past, since it gives an integrated picture with haemolymph volume about the effect of treatment. Since the total body water content could be partitioned into two fractions: tissue water and haemolymph water. The decrease in body weight of the Galleria larvae, pupae and adults postbacterial injection, as observed in the present study, may be attributed basically to the decrease of body water content. This decrease may be due to the loss of tissue water. These observations are in agreement with those of(Radwan,2015) working on $G$. mellonella and with those of Carrel et al. (1990); Bardoloi and Hazarika (1992); El-Kattan (1995) and Barakat and Meshrif (2007) who obtained similar results with other agents and other insect species.

With respect to sex, the fresh body weight of normal adult females was found to be much greater than males, i.e. the male moths displayed a greater loss of mass during the development in comparison to females. As there is no uptake of food during metamorphosis and the larval gut content has been purged prior to cocooning, so this difference in body mass is attributed to difference in excretion rate (Meylaers et al.,2007). When an infection occurs, the mass loss is affected in a sex-specific way, increasing in males over females. Since for female moths, it is important to retain as much mass as possible during development, as it is directly correlated with reproductive success (Honek, 1993; Nylin and Gotthard, 1998), but it is not the case in males. Similar observations on sexual dimorphism in mass loss were detected by Fischer and Fiedler (2000) in the Lepidopteran Lycaenatityrus and by Meylaers et al. (2007) on the same insect, G. mellonella infected by E. coli.

Concerning pupae coming from treated larvae and adult insects developed from previously challenged stages, no changes were detected in their body weights (wet and dry) and water content as compared with treated insects, but a significant decrease was found as compared with normal ones, indicating the appearance of a resistance transfer in the subsequent stages.

Many reports demonstrated that haemolymph is the water reservoir and that the haemolymph volume varies considerably with numerous factors such as age, developmental status, diet and hydration state (Edney, 1977; Barakat and Meshrif, 2007) with slight differences due to rearing conditions and diet.

An increase in blood volume was observed, in this study, in all tested developmental stages of Galleria following bacterial injection. It was obvious thus, that $\mathrm{Bt}$ decreased body water content, and in the same time, caused loss of water in the tissues (which was reflected from the decrease in the dry body weight) and gained it in the haemolymph. Similar results were obtained by (El-Kattan,1995) on Plodia interpunctella larvae and (Barakat,1997) on G. mellonella, larvae injected with different species of bacteria. Water withdrawal from the cells and tissues following bacterial infection may be due to the increase in the fluidity of the cell membrane (Bardoloi and Hazarika, 1992).

In contrast, some investigators including (Bucher,1957) on his study on Malacosoma pluviale infected with a spore-forming bacteria reported an extreme loss of water in the cells and tissues and subsequently in the haemolymph (i.e. decrease in the haemolymph volume). This is the case when bacteria invade the gut, producing diarrhea and/or vomiting. But in the present situation, when bacteria invade the hemocoel, diarrhea or vomiting was not observed and a water gain in the haemolymph was observed.

The current results also indicated that the haemolymph volume of normal 


\section{Physical variances in the hemolymph of Galleria mellonella L.(Lepidoptera: Pyralidae) following immune induction}

Galleria females is found to be greater than males this may be due to the increase of total haemocyte population of females over males as reported by (Sanjayan et al. 1996) worked on the milkweed bug, Spilostethus hospes. In addition, both males and females respond to the $\mathrm{Bt}$ infection by an increase in the haemolymph volume.

The haemolymph density and $\mathrm{pH}$ were also increased by the injection of bacteria into the different developmental stages of G. mellonella at almost all postinjection periods. This may be due to the decrease of blood volume as well as the increase of bacterial metabolites. These results are in agreement with those of Werner and Jones (1969), (Barakat,1997) on G. mellonella larvae and Angus and Heimpel (1956) on the larvae of Bombyx mori infected with Bacillus sotto.

\section{REFERENCES}

Angus, I.A. and Heimpel, A.M. (1956). An effect of Bacillus sotto on the larvae of Bombyx mori. Can. Entomologist, 88: 138-139.

Atmowidjojo A.H.; Erickson E.H.; Wheeler D.E. and Cohen A C. (1999). Regulation of haemolymph osmolality in feral and domestic honeybees Apis mellifera L. (Hymenoptera, Apidae). Comp. Biochem. Physiol., 122 (2):227233.

Barakat, E.M.S. (1997). A comparative study on the immune response of the wax moth, Galleria mellonella (L.) to some biotic and abiotic materials. Ph.D. Thesis, Ain Shams Univ. Egypt.

Barakat, E.M.S. and Meshrif, W.S. (2007). Physiological changes in the desert locust, Schistocerca gregaria F. in response to diet and injection with Bacillus thuringiensis. J. Environ Sci., 3: 210-239.

Bardoloi, S. and Hazarika, L.K. (1992). Seasonal Variations of Body
Weight, Lipid Reserves, Blood Volumes, and Hemocyte Population of Antheraea assama (Lepidoptera: Satumiidae). Environ. Entomol., 21(6): 13981403.

Brehelin, M. and Zachary, D. (1986). Insect haemocytes: A new classification to rule out the controversy. In: Immunity ininvertebrates. (Brehelin, M. Ed.). Pp. 36-46; Speringer-Verlag.

Bravo, A.; Gill, S.S. and Soberon, M. (2005). Bacillus thuringiensis: mechanisms and use. Comprehensive Molecc. Insect Sci.; 6:175-205.

Bucher, G.E. (1957).Disease of the larvae of tent caterpillars caused by a spore forming bacterium. Can. J. Microbiol., 3(5): 695-709.

Burges, H.D. (1978). Control of wax moth: Physical, Chemical and biological methods. Bee World, 59(4):129-138.

Campbell, J.J.R. and Konowalchuk, J. (1948). Comparisons of drop and pour plate counts of bacteria in raw milk. Can. J. Res. E., 26: 327-329.

Carrel, J.E.; Wood, J.M.; Yang, Z.; Mecairel, M.H. and Hindman, E.E. (1990). Diet, body water, and haemolymph content in the Blister beetle, Lytta polita (Coleoptera: Meloidae). Environ. Entomol., 19(5): 1283-1288.

Chapman, R.F. (2013). The insects: structure and Function. $5^{\text {th }}$ ed. Cambridge: Cambridge University Press, 109-132.

Edney, E.B. (1977). Water balance in land arthropods. In: Zoophysiology and ecology, (Hoar et al. eds.). Vol. 9.

El-Kattan, N.A.I. (1995). Physiological studies on the Indian meal moth, Plodia interpunctella HB. (Pyralidae: Lepidoptera) infected with microbial entomopathogens. 
Emad M.S. Barakat et al.

Ph.D. Thesis, Ain Shams Univ. Egypt.

Fischer, K. and Fiedler, K. (2000). Sex related differences in reaction norms in the butterfly Lycaena tityrus (Lepidoptera: Lycaenidae). Oikos, 90(2): 372-380.

Heimpel, A.M. (1955). The $\mathrm{pH}$ in the gut and blood of the larch sawfly, Pristiphora erichosonii (HTG.), and other insects with reference to the pathogenicity of Bacillus cereus. Can. J. Zool., 33: 99-106.

Honěk, A. (1993). Intraspecific variation in body size and fecundity in insects: a general relationship. Oikos, 483-492.

Jones, J.C. (1977). The circulatory system of Insecta, Charles C. Thomas, Springfield, I: 11.

Krishnendu, M.; Boran, A.; Torsten, H.; Eugen, D.; Andreas, V. and Trinad, C. (2010). Galleria mellonella as a model system for studying Listeria pathogenesis. Appl. Environment. Microbiol.,76(1): 310-317.

Kulkarni, N.; Kushwaha, D.K.; Mishra, V.K. and Paunikar, S. (2012). Effect of economic modification in artificial diet of greater wax moth Galleria mellonella (Lepidoptera: Pyralidae). Ind. J. Entomol.,74(4): 369-374.

Kurata, S.; Saito, H. and Natori, S. (1992). The $29 \mathrm{k}$ Dahemocyte proteinase dissociates fat body at metamorphosis of Sarcophaga. Developm. Biol., 153(1):115121.

Lee, R.M. (1961). The variation of blood volume with age in the desert locust, Schistocerca gregaria (Fosk.). J. Insect Physiol., 6: 36-51.

Lionakis, M.S. (2011). Drosophila and Galleria insect model hosts: new tools for the study of fungal virulence, pharmacology and immunology. Virulence, 2(6), 521527.

Mahmoud, D.M., Salem, D.A.M., Mo'men, S.A., Barakat, E.M.S. and
Salama, M.S. (2015). Purification and characterization of phenoloxidase from immunized haemolymph of Schistocerca gregaria. Afr. J. Biotechnol., 14(44): 3027-3036.

Meshrif, W.S. and Barakat, E.M.S. (2002). Cell mediated immunity in the Locust, Shistocerca gregaria against the bacterium, Bacillus thuringiensis. J. Egypt Acad. Soc. Environ. Developm., 2(1): 117130.

Meshrif, W.S., Barakat, E.M.S., Rohlfs, M., Shehata, M.G., Seif, A.I. and Hegazi, M.A.M. (2007). Characterization of extracellular enzymes and their role in studying the pathogenicity of the Hyphomycete fungi to the cotton leafworm, Spodoptera littoralis. Ain Shams Sci. Bull., 45: 1-16.

Meylaers, K.; Freitak, D. and Schoofs, L. (2007). Immunocompetence of Galleria mellonella: sex-and stagespecific differences and the physiological cost of mounting an immune response during metamorphosis. J. Insec. Physiol., 53(2): 146-156.

Miranpuri, G.S. and Khachatourians, G.G. (1993). Haemocyte surface changes in the migratory grasshopper, Melanoplus sanguinipes in response to wounding and infection with Beuveria bassiana. J. Entomol. Exp.,68: 157-164.

Momen, S.A.; Salem, D.A.M.; Barakat, E.M.S. and Salama, M.S. (2012). Activation of prophenoloxidase during bacterial injection into the desert locust, Schistocerca gregaria. Int. Sci Ind., 6(8): 496505.

Natori, S.; Shiraishi, H.; Hori, S. and Kobayashi, A. (1999). The roles of Sarcophaga defense molecules in immunity and metamorphosis, 


\section{Physical variances in the hemolymph of Galleria mellonella L.(Lepidoptera: Pyralidae) following immune induction}

Developm. Comp. Immunol., 23: 317-328.

Nylin, S. and Gotthard, K. (1998). Plasticity in life-history traits. Ann. Rev. Entomol., 43(1): 63-83.

Pereira, M.F.; Rossi, C.C.; Queiroz, M.V.; Martins, G.F.; Isaac, C.; Bosse, J.T.; Li, Y.; Wren, B.W.; Terra, V.S.; Cuccui, J.; Langford, P.R. and Bazzolli, D.M.S. (2015). Galleria mellonella is an effective model to study Actinobacillus pleuropneumoniae infection. $\mathrm{J}$. Microbiol., 161:387-400.

Radwan, M.H.I. (2015). Induced Immune Response against Bacillus thuringiensis in the Developmental Stages of the Greater Wax Moth, Galleria mellonella (Lepidoptera: Pyralidae). M.Sc. Thesis, Ain Shams Univ. Egypt.

Rogoff, M.H.; Ignoffo, C.M.; Singer, S. and Prieto, A.P. (1969). Insecticidal activity of 31 strains of Bacillus against five insect species. J. Invertebr. Pathol..14: 122-129.

Sanjayan, K.P.; Ravikumar, T. and Albert, S. (1996). Changes in the haemocyte profile of Spilostethus hospes(fab.);

(Heteroptera:

Lygaeidae); in relation to eclosion, sex and mating. J. Biosci., 21(6) 781-788.
Schmid-Hempel, P. (2003). Variation in immune defence as a question of evolutionary ecology. Proc. R. Soc. B, 270: 357-366.

Schmid-Hempel, P. (2005). Evolutionary ecology of insect immune defenses. Annual Rev Entomol., 50: 529551.

Scully, L.R.; and Bidochka, M.J. (2006). Developing insect models for the study of current and emerging human pathogens. FEMS microbiology letters,263(1): 1-9.

Sheldon, B.C. and Verhulst, S. (1996). Ecological immunology: costly parasite defences and trade-offs in evolutionary ecology. Trends Ecol. Evol., 11: 317-321.

Werner, R.A. and Jones, J.C. (1969). Phagocytic haemocytes in unfixed Galleria mellonellalarvae.J. Insect Physiol., 15(3): 425-437.

Wojda, I. (2017). Immunity of the greater wax moth Galleria mellonella: Insect Science, 24(3): 342-357.

Wigglesworth, V.B. (1974). Insect physiology. $7^{\text {th }}$ edition.

Yeager, J.F. and Munson, S.C. (1950). Blood volume of the roach, Periplaneta americana determined by several methods. Arthropoda,1: 255-256. 